\title{
LAS POLÍTICAS DE EVALUACIÓN UNIVERSITARIA EN ARGENTINA: BALANCE Y DESAFÍOS
}

\section{University Evaluation Policies in Argentina: Balance and Challenges}

As políticas de avaliação universitária na Argentina: balanço e desafíos

RECIBIDO: 15 DE DICIEMBRE DE 2015

María Catalina Nosiglia (Argentina) catinosiglia@gmail.com

Licenciada en Ciencias de la Educación

Universidad de Buenos Aires

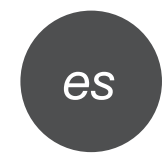

\section{RESUMEN}

El objetivo de este artículo es presentar algunas reflexiones sobre las tensiones de las políticas de evaluación universitaria, distinguiendo analíticamente cuatro cuestiones que atraviesan este campo y que plantean relaciones complejas: evaluación y autonomía, evaluación y planeamiento, evaluación y calidad, evaluación y pertinencia social.

Asimismo, se incluyen algunas características de la conformación del campo de la evaluación y acreditación universitaria, haciendo especial referencia al caso de la Universidad de Buenos Aires. Finalmente, se plantean consideraciones para futuras investigaciones y revisiones de esta política, tras dos décadas de implementación.

\section{EVALUADO:11DE ABRILDE2016}

\section{ACEPTADO:20 DE MAYO DE 2016}

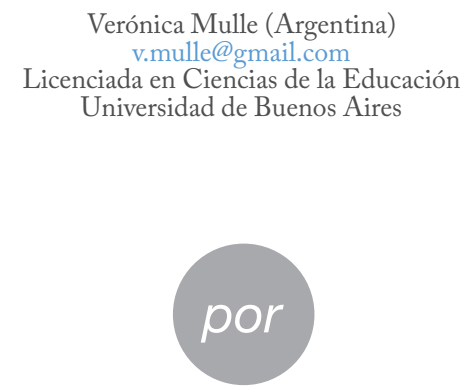

\section{en}

ABSTRACT

This article aims to present some reflections regarding the tensions of university evaluation policies by highlighting four issues in this field that suggest complex relationships: evaluation and autonomy, evaluation and planning, evaluation and quality, and evaluation and social relevance.

Likewise, we include some characteristics of evaluation and university certification field setup by making special reference to the case of the University of Buenos Aires. Finally, we suggest ideas to keep into account in future researches as well as revisions to this policy that has been implemented for more than two decades.

\section{RESUMO}

O objetivo deste artigo é apresentar algumas reflexões sobre as tensões das políticas de avaliação universitária, distinguindo analiticamente quatro questões que atravessam este campo e que apresentam relações complexas: avaliação e autonomia, avaliação e planejamento, avaliação e qualidade, avaliação e pertinência social.

Mesmo assim, incluem-se algumas características da conformação do campo da avaliação e acreditação universitária, fazendo especial referência ao caso da Universidade de Buenos Aires. Finalmente, apresentam-se considerações para futuras pesquisas e revisões desta política, depois de duas décadas de implementação.
PalabRas CLAVE: educación, evaluación universitaria, universidades, política educativa, autoevaluación instituciona.
KEYWORDS: Education, university evaluation, universities, education policy, institutional self-evaluation.
PALAVRAS CHAVE: educação, avaliação universitária, universidades, política educativa, auto avaliação institucional. 


\section{INTRODUCCIÓN}

La evaluación educativa fue una de las políticas más sobresaliente de la década de 1990 en Argentina. Esta temática estuvo presente en la agenda de las políticas neoliberales de todos los niveles educativos, asociada a una cuestión más amplia: la preocupación por la calidad de la educación. Las orientaciones políticas en esta materia suponían la necesidad de competencia interinstitucional, la mayor responsabilidad de las instituciones y los docentes por los resultados y la rendición de cuentas como mecanismos para garantizarla.

En las universidades argentinas, la introducción de los mecanismos de evaluación institucional y la acreditación de carreras de grado y posgrado fueron establecidos en 1995 a partir de la Ley de Educación Superior 24521.

Esta política fue una de las cuestiones más controvertidas de la década de 1990 — junto con otras, como la de incentivos a los docentes investigadores - y a la vez una de las que más impacto tuvo en la cultura académica de las instituciones. Generó la necesidad de modificaciones en lo curricular, en la infraestructura y, en alguna medida, tuvo efectos sobre la carrera académica. El impacto se puede medir tanto respecto de su permanencia como de su alcance territorial, que abarca lo nacional, lo regional y lo internacional.

Las nuevas regulaciones en esta materia fueron inicialmente cuestionadas y resistidas por la mayor parte de las instituciones universitarias en Argentina, ya que, por un lado, se las consideraba un avance sobre la autonomía, en la medida en que sometía las prácticas institucionales y académicas de la universidad a un proceso de evaluación basado en estándares y en una agencia externa a las universidades; $y$ por otro, por el temor de que se intro-

Panorama I pp. 33-44 I Volumen 101 Número 19। Julio-diciembre 2016 | Sin embargo, paulatinamente, a partir de políticas de incentivos implementadas por la Secretaría de Políticas Universitarias, los contenidos de los fallos judiciales sobre la inconstitucionalidad de la Ley de Educación Superior — que fueron adversos para la mayoría de las universidades- y por la adaptación de las instituciones a esta política, se la fue aceptando y un número significativo de docentes e investigadores participaron como pares evaluadores en la implementación de estos procesos.

Ya sea por adhesión a esta política, por la obtención de incentivos de orden simbólico y material o con una aceptación crítica, la mayoría de las universidades se fueron involucrando en estos procesos. Esta posición, si bien goza de cierta estabilidad, aún continúa siendo rechazada activamente por algunos sectores, principalmente del claustro estudiantil.

El objetivo de este trabajo es presentar algunas reflexiones sobre las tensiones de las políticas de evaluación universitaria y distinguir analíticamente cuatro cuestiones que atraviesan este campo y que plantean relaciones complejas: evaluación y autonomía, evaluación y planeamiento, evaluación y calidad, evaluación y pertinencia social.

Asimismo, se incluyen algunas características de la conformación del campo de la evaluación y acreditación universitaria en Argentina, haciendo especial referencia al caso de la Universidad de Buenos Aires. Finalmente, se plantean consideraciones para futuras revisiones de esta política, tras dos décadas de implementación de mecanismos de evaluación universitaria en este contexto.

\section{TENDENCIAS RECIENTES DEL SISTEMA DE EDUCACIÓN SUPERIOR}

A modo introductorio de las cuestiones teóricas que se tratarán más adelante, dedicaremos este apartado a caracterizar brevemente algunas tendencias recientes del sistema de educación superior, que dan cuenta del escenario en el que se implementan las políticas de evaluación.

En un estudio reciente, Brunner y Villalobos (2014) analizan las tendencias de la educación superior en Iberoamérica y observan como rasgo principal la expansión de la matrícula de este nivel. Durante el último lustro (2005-2011), la población estudiantil se incrementó de 18.4 millones a casi 25 millones, siguiendo una tendencia sostenida de crecimiento desde la década de 1970. En muchos países de la región latinoamericana, esta 
expansión fue absorbida principalmente por el sector privado, por ofertas de variable calidad. En algunos países, la matrícula del sector privado supera considerablemente la del sector público.

En Iberoamérica, hay 15214 instituciones de educación superior, de las cuales $24 \%$ son universidades u organizaciones equivalentes - $69 \%$ de estas es de gestión privada- y $76 \%$ instituciones no universitarias (Brunner y Villalobos, 2014).

En Argentina, tanto el sector público como el privado han crecido desde la década de 1990, con una tendencia a la desregulación de la oferta universitaria privada y más contemporáneamente con la creación de más de una docena de universidades públicas. Actualmente en Argentina contamos con 135 instituciones universitarias y 2213 instituciones de nivel superior no universitarias. ${ }^{1}$ Entre 1995 y 2015, la cantidad de universidades creció más de $60 \%$, y fue mayor el crecimiento de instituciones públicas (81\%) que el de las privadas (51\%) en todo el periodo. Solo en la última década se crearon 17 nuevas universidades nacionales y 7 universidades privadas.

Más allá del desarrollo particular de cada sector respecto de la cantidad de instituciones, el número total de universidades públicas y privadas es semejante ${ }^{2} \sin$ embargo, la matrícula universitaria se concentra principalmente en el sector público (79\%) mientras solo $21 \%$ concurre a establecimientos privados. Esta distribución de la matrícula por sector ha sido similar durante los últimos veinte años, aunque en términos relativos el crecimiento de la matrícula privada fue mayor que el de la pública.

Por otra parte, las instituciones se han diversificado. En primer término, por las funciones que desarrollan las universidades, la mayor parte realiza solo docencia, una menor parte investigación y docencia. En segundo término, porque las ofertas académicas son variadas,

1 Para el caso de las instituciones universitarias, este dato fue construido a partir de las normas de creación de las instituciones y corresponde a 2015, mientras que la información de las instituciones de nivel superior no universitario corresponde a 2014 y proviene del anuario estadístico elaborado por la Dirección Nacional de Información y Evaluación de la Calidad Educativa publicado en 2015

2 En el caso de las instituciones universitarias, $51 \%$ son instituciones públicas y el resto privadas. Si se observa el sector de instituciones de educación superior no universitarias, la distribución varía un poco: el sector público concentra $46 \%$ de las instituciones, mientras que $54 \%$ son privadas. algunas con gran desarrollo de posgrados y otras atienden a formaciones de pregrado y grado exclusivamente. En tercer término, por sus perfiles, algunas son de élite y otras promueven la inclusión de los recientes egresados de la secundaria, que a su vez son la primera generación familiar que asiste a la universidad.

Asimismo se incrementó la oferta de posgrados. En Argentina, su cantidad se triplicó en solo dos décadas. ${ }^{3}$ Este crecimiento se dio, inicialmente, con escasas regulaciones por parte del Estado. La expansión de los posgrados responde a la necesidad de actualización permanente y mayores exigencias en la carrera académica de los docentes.

También se produjeron cambios en los modos de enseñar y aprender, que implicaron un pasaje progresivo desde los modos convencionales de enseñanza, centrados en la clases presenciales expositivas, a nuevos modos promovidos por el campo experimental que provee el uso de las tecnologías de la información y de la comunicación (TIC), que asumen nuevas prácticas docentes, nuevos espacios y conceptos de aprendizaje, nuevas formas de organización, comunicación y gestión del conocimiento y su vínculo con las ocupaciones y la vida cotidiana.

Otra tendencia del sistema de educación superior se refiere al incremento de la internacionalización de la educación superior, lo cual se expresa en el creciente flujo de la movilidad internacional de estudiantes, en el aumento de intercambios e interacción de académicos e investigadores de diferentes partes del mundo a través de redes globales, en la convergencia estandarizada de programas y carreras y en el comercio de servicios trasnacionales de educación superior. Algunos indicadores que reflejan estas tendencias es la cantidad de estudiantes internacionales, los acuerdos de reconocimiento mutuo de estudios, la cantidad de redes y asociaciones de universidades que se han desarrollado, así como los diferentes programas de movilidad y los convenios de cooperación académica. Por último, la oferta trasnacional de educación superior, apoyada por las oportunidades que brindan las TIC, también generó nuevos formatos institucionales con ofertas de formación masivas y desarrolladas íntegramente bajo la modalidad virtual.

\footnotetext{
3 Los datos se construyeron a partir de dos fuentes: De la Fare y Lenz (2010) y Coneau (2015).
} 
María Catalina

Nosiglia I

Verónica

Mulle I

En último término, como sustrato a todos los cambios descritos, se ha transformado la relación entre el Estado, la sociedad y las universidades, lo cual constituye una de las piedras angulares de la instalación de mecanismos de evaluación universitaria, que se basan en lo que algunos autores denominaron la construcción de un "nuevo contrato social" entre las universidades y el Estado (Brunner, 1993). Este nuevo contrato supone la sustitución del débil control administrativo del Estado por la evaluación, el cambio de criterios en la asignación de recursos públicos basado en objetivos y metas convenidos y un compromiso por parte de las universidades de diversificar sus fuentes de financiamiento.

El pasaje del Estado benevolente al Estado evaluador (Neave y Van Vught, 1991) es parte de una reforma más amplia de los organismos públicos que tiene fundamentos en la teoría del New Public Management. Esta perspectiva supone una visión competitiva de las instituciones que tiene efectos en la forma en que se define el gobierno de los sistemas y de las instituciones de educación superior en su interior (Dobbins, Knill y Vögtle, 2011; Kretek, Dragsic y Kehm, 2013). Para adecuarse a este escenario de competencia interinstitucional, las universidades requieren tener una gestión institucional más jerárquica y profesionalizada, una mayor eficiencia organizativa y un mayor grado de responsabilidad hacia el público (rendición de cuentas).

En síntesis, las tendencias observadas, así como las políticas promovidas, constituyeron el escenario en el que se desarrollaron - y aún hoy continúan desarrollándoselos procesos de evaluación y acreditación universitaria.

Aunque en la actualidad se ha superado la resistencia inicial que generaban estos procesos, la evaluación universitaria no está exenta de tensiones. A continuación, se tratan algunas de ellas.

Panorama I

$\mathrm{El}$ análisis de las tensiones presentes en las políticas de evaluación universitaria que se propone distingue analíticamente cuatro cuestiones que atraviesan este campo y que plantean relaciones complejas: evaluación y autonomía, evaluación y planeamiento, evaluación y calidad, evaluación y pertinencia social.
La definición de estas cuestiones incluye problemas de diferente índole: algunas son antinomias (contradicciones entre dos principios racionales), otras son dilemas (alternativas frente a una situación y que se presentan simultáneamente) o falsas premisas (enunciados que estimados como ciertos conducen a error).

\section{EVALUACIÓN Y AUTONOMÍA}

Tanto la autonomía como la evaluación son términos polisémicos y es necesario explicitar su acepción en cada caso.

La evaluación es entendida como el proceso de recolección de información y a partir de ella la construcción de juicios de valor. Las unidades de evaluación pueden ser de muy diversa índole: desde el sistema educativo, una institución hasta los actores que participan del proceso educativo: alumnos y docentes. Además, siguiendo a Camilloni (2004), la evaluación puede tener distintos objetivos: puede ser diagnóstica, cuando se quiere conocer las causas de determinado fenómeno; preventiva, cuando se recoge información frente a una situación hipotética que se quiere impedir; prospectiva $-\mathrm{y}$ en parte toda evaluación lo es- en cuanto la información $\mathrm{y}$ los juicios que se realizan sobre una situación actual pueden ser utilizados para hacer predicciones respecto del futuro; puede ser sumativa cuando toma los resultados de un proceso y analiza si eso estuvo bien o estuvo mal; o formativa, cuando algo está en proceso y nosotros recogemos información para ir estableciendo medidas de corrección para mejorar el resultado de ese proceso. También puede ser reguladora, cuando se analiza una situación determinada para tomar las medidas necesarias para corregir o para que sigan así adelante; también puede ser justificativa o acreditadora cuando un grupo externo va a recibir información sobre algo y a emitir un juicio de valor que es público. Hay también una evaluación de costo-efectividad, que relaciona los recursos utilizados y los resultados obtenidos (Camilloni, 2004). Además, difieren los propósitos de la evaluación según lo que se espera producir (recomendaciones, directivas, explicaciones o diferentes puntos de vista dentro de la comunidad académica, por ejemplo) y los actores que la realizan (externa o autoevaluación o una combinación de ambas).

La definición de la autonomía universitaria, por otra parte, resulta compleja por la historicidad de este 
concepto que nos retrotrae hasta los tiempos medievales de los "fueros" de la universidad, su esfera de protección frente a la arbitrariedad del poder, por sus diferentes interpretaciones jurídicas y por las diferentes dimensiones a las que se aplica (institucional, académica, administrativa financiera).

Históricamente en Argentina los alcances y las limitaciones de la autonomía universitaria tuvieron una base legal, es decir, estuvieron sujetos a las características que establecían las leyes emanadas por el Congreso a partir de lo estas pautasen. En 1994, esto cambia por cuanto los principios de autonomía y autarquía son incorporados como garantías de las universidades nacionales en la Constitución Nacional.

Si bien la evaluación y la autonomía universitaria no constituyen términos opuestos en sí mismos, entran en tensión cuando se les otorga la función de evaluar un agente externo a la propia universidad y según criterios construidos por otros organismos.

Precisamente con la sanción de la Ley de Educación Superior 24521 en 1995, se introducen procesos de evaluación y acreditación universitaria con carácter sistemático y en todo el sistema de educación superior. Estos procesos son llevados adelante por la Comisión Nacional de Evaluación y Acreditación Universitaria (Coneau), un organismo descentralizado, que funciona en jurisdicción del Ministerio de Educación y que tiene como funciones principales coordinar y llevar adelante la evaluación externa de las universidades, evaluar los proyectos institucionales de universidades nuevas públicas y privadas y acreditar carreras de grado y de posgrado.

En un trabajo reciente, Araújo (2015) señala justamente que en Argentina conviven dos enfoques de evaluación: por un lado, un enfoque comprensivo y centrado en el reconocimiento de las particularidades de los proyectos institucionales de los establecimientos que se refleja en la evaluación institucional; por el otro, un enfoque de carácter comparativo, que se observa en la acreditación de carreras de grado y posgrado, en la medida en que cada oferta de formación — que estuviera alcanzada por estos procesos - se compara con referencias externas a la propia carreras, a través de criterios o estándares definidos previamente.
Desde un punto de vista se puede sostener que el principio de autonomía universitaria se encuentra resguardado, ya que, en primer lugar, tanto los informes de acreditación como los de evaluación externa parten de autoevaluaciones realizadas por las propias instituciones; en segundo lugar, ambos procesos se apoyan en los juicios de pares académicos (docentes e investigadores de las propias universidades especialistas en las disciplinas). Asimismo, en relación con la evaluación institucional no existen patrones o estándares técnicos a los que las universidades deban ajustarse y por cuyo incumplimiento pudieran ser reprendidas o censuradas. La evaluación se centra en la valoración de la idiosincrasia del proyecto universitario propio. Distintos autores coinciden en señalar que la evaluación externa es un proceso que contribuye a mirar la institución como un todo, por encima de las múltiples culturas disciplinares que la conforman, dado su enfoque holístico y sociohistórico y, en este sentido, morigera la percepción de los actores respecto de la universidad como federación o conjunto de facultades (Araújo, 2015; Stubrin, 2014).

Con respecto a la acreditación de carreras, esta se basa en la aplicación de ciertos estándares de calidad. Los estándares son aprobados por el Ministerio de Educación a propuesta del Consejo de Universidades, ${ }^{4}$ según lo establece la normativa, pero parten de propuestas que son elaboradas por las propias universidades a través de asociaciones de decanos o de facultades y consensuadas en el Consejo Interuniversitario Nacional (compuesto por los rectores de universidades nacionales). Asimismo, la nómina de expertos a partir de la que la Coneau designa los comités de pares que emiten los dictámenes se elabora con las propuestas de las universidades.

Desde otra perspectiva, se sostiene que los procesos de evaluación institucional estuvieron sesgados a aquellas cuestiones que se suponía se iban a evaluar, y se aprendió a presentar la información de modo de ocultar o disminuir las posibles críticas sobre cuestiones problemáticas de las instituciones. En el caso de las acreditaciones de carreras de grado, algunos sostienen que llevó a la homogeneización de los planes de estudios y que la intromisión de las corporaciones en la fijación de extensas actividades reservadas condujo a una ampliación de las materias y los contenidos.

\footnotetext{
4 El Consejo de Universidades está integrado por rectores de universidades nacionales, rectores de universidades privadas y el ministro de Educación de la Nación, quien preside.
}

Las políticas de evaluación universitaria en Argentina: balance y desafíos
I Panorama | pp. 33-44 I Volumen 10 I Número 19 I Julio-diciembre I 2016 
María Catalina

Nosiglia I

Verónica

Mulle I

Detrás de estas cuestiones, esta tensión convive entre la libertad de las universidades para definir diferentes aspectos de su vida institucional (académica, administrativa, etc.) y la función del Estado de velar por el interés público regulando la actividad de estos establecimientos.

\section{EVALUACIÓN Y PLANEAMIENTO}

La relación entre evaluación y planeamiento resulta en parte evidente, puesto que el planeamiento parece poco viable si no parte de algún tipo de evaluación. En general, la evaluación es concebida como una herramienta de gestión vinculada a los procesos de planificación de las instituciones, con la finalidad de contribuir al mejoramiento.

Pero también, siguiendo la perspectiva de algunos organismos, el planeamiento institucional puede ser considerado un indicador para la evaluación de la calidad de la educación superior. Específicamente, el Centro Interuniversitario de Desarrollo considera que la evaluación de la calidad sería posible en la medida en que las instituciones universitarias formulan su proyecto institucional y sus planes de corto, mediano y largo plazo, para cumplir las metas que se proponen. Esta perspectiva se basa en la idea de que la calidad es relativa y

el referente está establecido por la propia institución cuando define qué va a hacer, es decir, cuando define su misión, objetivos, metas y estrategias aun cuando pueda haber aspectos en los que será necesario atenerse a

Panorama I pp. 33-44। Volumen 10 I Número 19। Julio-diciembre 2016 I relaciones entre autonomía, planeamiento y políticas públicas,

la formulación y ejecución de planes es utilizada, entonces, para contrarrestar o superar algunos vicios típicos en que puede recaer cualquier administración: en primer lugar, el tradicionalismo, una manera de hacer que perpetúa el pasado; en segundo lugar, la rutinización, una reproducción de prácticas cuyo sentido originario se ha olvidado; por último, el "presente perpetuo", entendido como la tendencia a vivir día por día, prescindiendo de la reflexión tanto sobre el pasado como sobre el futuro (2014, p. 51).

En este sentido, la evaluación universitaria podría contribuir al planeamiento en dos niveles: en el sistema universitario, si el Estado se sirve de la información que suministran las evaluaciones para orientar las políticas públicas según objetivos fijados; en cada universidad, a partir de la autoevaluación y los informes de evaluación externa y las recomendaciones al planeamiento universitario que allí se incluyen.

Las políticas de evaluación parecen contener el potencial para operar en favor del planeamiento si fuera utilizada por las propias universidades para la toma de decisiones en el ámbito institucional, por un lado, y en el ámbito interinstitucional, por otro, sirviéndose de las instancias de coordinación sistémica. Sin embargo, la vinculación y retroalimentación entre estos procesos no se encuentra sumamente desarrollada, en especial en lo interinstitucional.

\section{EVALUACIÓN Y CALIDAD}

Como se ha mencionado más arriba, la evaluación universitaria es un concepto que se instala en el debate político dentro de una discusión más amplia, cuyo foco está puesto en la calidad. Si bien el tema de la calidad estuvo siempre presente en la discusión de las políticas académicas de la comunidad universitaria, en especial desde los profesores en sus tareas de enseñanza e investigación con escasa intervención por parte de actores externos, a partir de la década de 1990 la calidad se asocia con el tema de la evaluación en todos los niveles de enseñanza. Esto mismo también fue promovido por la intervención de agencias nacionales e internacionales, que desarrollaron pruebas para la evaluación de aprendizajes o competencias (como las pruebas PISA [Programme for International Student Assessment]), cuyos resultados suelen tener repercusiones importantes en 
los medios masivos de comunicación y ejercen presión sobre los Gobiernos.

Sin embargo, el concepto de calidad es polisémico y multidimensional. En este sentido, su carácter relativo está atravesado por los actores que lo definen, el momento histórico en que lo hacen, el espacio geográfico en el que se encuentran y las corrientes ideológicas desde las que se posicionan. Es un concepto esencialmente político, ya que surge de los intereses de los diferentes actores subordinado a concepciones ético-políticas. Los sentidos se producen en un espacio social de disputas de poder. En consecuencia, la forma en que se concibe la universidad influye directamente en el modo en que pensamos la calidad, cómo alcanzarla, mejorarla y garantizarla.

Por ello, el concepto de calidad es una construcción social, que varía según los intereses de los grupos de dentro y de fuera de la institución educativa, que refleja las características de la sociedad que se desea para hoy y que se proyecta para el futuro. No es un concepto unívoco y fijo, sino que debe ser construido a través de consensos y negociaciones entre los actores (Dias Sobrihno, 2003).

La idea de calidad remite a cuestiones diferentes según los actores que la definen: para los académicos se refiere a los saberes; para los empleadores, a competencias; para los estudiantes, a la empleabilidad; para la sociedad, a ciudadanos respetables y competentes; para el Estado, según la concepción que asuma, puede variar de aspectos vinculados con el desarrollo social y humano a la eficiencia, a los costos y a los requerimientos de capital humano (Fernández Lamarra, 2008).

Como se observa, más allá de las distintas definiciones, se acuerda que la calidad de la educación superior debe incluir y articular las visiones y demandas de distintos actores, con los valores y propósitos de las instituciones universitarias y de la sociedad.

El tipo de evaluación y acreditación universitaria que se desarrolló en Argentina respondió en un principio al modelo de evaluación para el control, con la acreditación de las carreras de grado y las de posgrado que incluyen una calificación. Sin embargo, luego de las primeras rondas de evaluación y acreditación de instituciones y carreras, se vio la necesidad de que, si se quería contribuir a mejorar su calidad y superar los problemas detectados en los procesos de evaluación, se debían desarrollar nuevos instrumentos de políticas para el aseguramiento de la calidad a través de programas y fondos especiales (Nosiglia, 2008).

Así como la evaluación quedó asociada a la mejora de la calidad, también la idea de calidad fue vinculada al concepto de pertinencia como desafíos para las universidades, como parte de la agenda para el sector promovida por algunos organismos internacionales (Unzué, 2014). Sin embargo, esta asociación no es necesariamente evidente, ya que puede haber actividades de calidad que no sean pertinentes, $y$ actividades pertinentes que no reúnan los requisitos mínimos de calidad. Justamente la tensión entre evaluación y pertinencia social será la que abordaremos a continuación.

\section{EVALUACIÓN Y PERTINENCIA SOCLAL}

La evaluación fue asociada a la pertinencia en cuanto, como sostienen diversos autores, la evaluación debe producir significados sobre cómo las instituciones educativas están cumpliendo las funciones sociales de formación humana integral y de producción de conocimientos importantes, para la solución de problemas de las comunidades y la construcción de la nación (Dias Sobrinho, 2012).

El concepto de pertinencia es propio del ámbito organizacional y su protagonismo en las últimas décadas es parte de una transformación más amplia, que se vincula a la redefinición de las relaciones entre la sociedad y la universidad.

La reconfiguración global de las universidades produjo efectos en la cultura académica, y la emergencia de la noción de pertinencia comienza a reemplazar las tradicionales ideas de sentido, misión o función social y se convierten en el concepto privilegiado para analizar las universidades en diversos registros: curricular, institucional, sistémico, disciplinar, etc. (Naidorf, Giordana y Horn, 2007).

La noción de pertinencia de la educación superior (en francés: pertinence, en inglés: relevance) es introducida por documentos especializados de la Unesco desde 
María Catalina

Nosiglia I

Verónica

Mulle I

1995, y es reforzada sistemáticamente a partir de 1998, en ocasión de la Conferencia Mundial sobre la Educación Superior en el siglo XXI. En los documentos, se la define de la siguiente manera: "La pertinencia de la educación superior debe ser considerada esencialmente en función de su lugar y su rol en la sociedad, es decir, su misión en materia de educación, de investigación y de servicios, tanto como sus lazos con el mundo del trabajo en el sentido más amplio, de sus relaciones con el Estado y las fuentes del financiamiento público y de sus interacciones con los otros grados y formas de enseñanza” (Unesco, 1998, p. 31).

La categoría de pertinencia utilizada desde esta perspectiva procura contrarrestar dos aspectos: por una parte, la tendencia al autoencierro de las instituciones universitarias, favorecida por una comprensión demasiado unilateral de la autonomía académica; y por otra, la tendencia de una agenda académica definida desde parámetros puramente disciplinares y, por ende, desconectados - se supone- de las realidades y los contextos sociales (locales, nacionales, regionales o globales).

A partir de la década de 1980 y principalmente de la década de 1990, se desplazó el eje desde el plano de la responsabilidad autónoma de la universidad al plano de su hibridación con la sociedad y el mercado, tanto en la cultura académica como en la evaluación de la pertinencia social universitaria. En este sentido, la responsabilidad se vio transformada en accountability.

Las diversas perspectivas que hacen referencia a modelos distintos y contradictorios de universidad también ensayan definiciones diferentes sobre el término pertinencia.

Desde un enfoque economicista se considera que un plan de estudios, una investigación o una institución cumplen con parámetros de pertinencia social cuando responden a demandas del mercado, es rentable y su producción es eficiente.

Volumen 10 I Número 19। Julio-diciembre 2016 I

Desde una perspectiva social o integral, las mismas actividades que se realizan en la universidad son pertinentes cuando logran vincularse e integrarse con la sociedad de la cual forman parte y en la que contribuyen desde su especificidad al mejoramiento de las condiciones de vida — en sentido amplio— de las mayorías, desde una visión de largo plazo y de manera sustentable. En algunas circunstancias, lo anterior se cumple a través de la elaboración de herramientas teóricas que permiten comprender y transformar las condiciones actuales y rescatar el valor de la impertinencia epistémica.

\section{ALGUNAS CARACTERÍSTICAS DE LA CONFORMACIÓN DE LA EVALUACIÓN UNIVERSITARIA EN ARGENTINA CON REFERENCIA A LA UNIVERSIDAD DE BUENOS AIRES}

En Argentina, la preocupación por la evaluación y acreditación del sistema universitario tiene antecedentes concretos hacia fines de la década de 1980. Algunos autores plantean diferentes etapas en la conformación de este campo según las posiciones político-académicas adoptadas por parte de las instituciones universitarias. En este sentido, es posible distinguir tres momentos diferentes en la estructuración de esta relación: 1) las universidades presentan una posición crítica a la evaluación, inicialmente como resistencia y luego como contrapropuesta, 2) el Poder Ejecutivo se torna hegemónico en la producción de propuestas y se instala una regulación heterónoma de los mecanismos de evaluación en el ámbito universitario y 3) las instituciones universitarias adoptan los procesos definidos y las prácticas de evaluación y acreditación que se incorporan como procesos de rutina (Mollis, 1999; Krotsch, 2001).

El primer momento, entre 1987 y 1992, cuando con el objetivo de promover el financiamiento de la coordinación y gestión de las universidades nacionales se implementó un proyecto acordado entre el Ministerio de Educación y Justicia y el Banco Mundial. En 1991, el Gobierno firma un acuerdo con el Consejo Interuniversitario Nacional con el objetivo de ejecutar el Programa de Fortalecimiento a la Gestión y Coordinación Universitaria, conocido como Subproyecto 06, que contenía un componente vinculado a la evaluación de la calidad. Este componente fue materia de acuerdo y negociación entre el Estado y las universidades.

En este periodo, la Universidad de Buenos Aires también manifestaba la preocupación por la evaluación de la calidad educativa, a través de la creación de diversos programas orientados a evaluar diferentes componentes de la actividad académica. Por ejemplo, en 1988, se creó el programa de Evaluación del volumen y la calidad de la oferta educativa de la Universidad de Buenos Aires en relación con la demandas de la sociedad; más tarde, por 
iniciativa de la Secretaría de Asuntos Académicos, se inició una tarea de evaluación de los programas de posgrado; en 1991, se creó un programa de supervisión y evaluación de la gestión docente y de capacitación y perfeccionamiento del personal encargado de su gestión. Y, en 1995, se propuso una reforma de la universidad y los diversos aspectos de la vida académica (currículo, investigación, extensión y transferencia y estructura académica), con el objetivo de elevar la calidad y la pertinencia de los aportes de las actividades de la universidad frente a las nuevas demandas y transformaciones sociales, que se plasmó en el documento de base Propuesta para el cambio de la Universidad de Buenos Aires, elaborado en Colón (Entre Ríos), el mismo año. Específicamente, en relación con la evaluación universitaria, se plantea el problema de la falta de evaluación integral de las actividades y la necesidad de crear instancias y normas para su evaluación, e incluso unidades de control de gestión permanente para el seguimiento y la evaluación de las estructuras académicas. Estas tareas fueron discontinuadas en el tiempo, cuando algunas unidades académicas decidieron participar en la acreditación de carreras de grado o posgrado.

El segundo momento, caracterizado por la hegemonía del Poder Ejecutivo y la regulación heterónoma de los mecanismos de evaluación en el ámbito universitario, se inicia con la creación de la Secretaría de Políticas Universitarias en 1993, que produjo convenios con universidades para realizar evaluaciones internas y externas. Con la sanción de la Ley de Educación Superior, se cristaliza en un cuerpo normativo todo un conjunto de políticas que venían desarrollándose en un mismo sentido. La relación entre el Gobierno nacional y las universidades nacionales, en el periodo inmediato a la sanción de la Ley de Educación Superior, estuvo permeada por la resistencia de las instituciones a la aplicación de algunas disposiciones contenidas en la norma. Un ejemplo de esta postura lo constituyen los diversos recursos que varias universidades presentaron en la Justicia,${ }^{5}$ en los que uno de los temas cuestionados fue el establecimiento de los procesos de evaluación y acreditación periódicos y la creación de un organismo externo a las universidades al que se le confería el desarrollo de estas tareas. Los fallos fueron adversos a las universida-

\footnotetext{
5 Se trata de las presentaciones efectuadas por las universidades Nacionales del Centro de la Provincia de Buenos Aires, de Entre Ríos, de Luján, de Rosario, del Litoral, de Córdoba, de San Luis, de San Juan, de Catamarca, de Jujuy, de Misiones, de La Pampa, de Salta, de Río Cuarto, de Mar del Plata, la Tecnológica Nacional y la Universidad de Buenos Aires.
}

des, excepto el fallo del juez Marinelli en 1996 para el caso de la Universidad de Buenos Aires.

El fallo del juez Marinelli dio lugar a la solicitud de la Universidad de Buenos Aires de inconstitucionalidad de la Ley de Educación Superior solo parcialmente. Entre los artículos que consideró inconstitucionales se encuentran los artículos 42, 43 y el inciso b del artículo 46 de la actual Ley de Educación Superior, relativos a los títulos habilitantes y su evaluación y acreditación. De este modo, la Universidad de Buenos Aires quedó eximida de acreditar sus carreras de grado y posgrado.

El tercer momento que se señala (1997-2013) se basa en la adopción por parte de las instituciones universitarias de los procesos definidos y la rutinización de las prácticas de evaluación y acreditación. El estudio de las prácticas institucionales desarrolladas en el proceso de evaluación y acreditación en la actualidad muestra que la evaluación se ha incorporado como una práctica habitual, debido a los instrumentos, procedimientos, tiempos y factores políticos que la atraviesan.

La Universidad de Buenos Aires tuvo una posición cambiante a lo largo del tiempo. En 1997, el Consejo Superior hizo una declaración en la que autorizaba, en los casos que se considere conveniente, la presentación voluntaria a las evaluaciones y la acreditación de las carreras de posgrado ante la Coneau, aclarando que no implicaba la renuncia a la plena vigencia de la autonomía universitaria, por haber sido de carácter voluntario. ${ }^{6}$ Esta postura cambió en 2000, cuando este mismo órgano consideró que no se contaban con las garantías mínimas para someterse a los dictados de la Coneau, a pesar de que múltiples posgrados ya habían sido acreditados y evaluados con las más altas calificaciones, y resolvió suspender su presentación ante la Coneau. Un año después, se autorizó a todas las unidades académicas a concurrir a las convocatorias para la evaluación y acreditación de los posgrados.

En la actualidad, y como consecuencia de los cambios de posición frente a la Coneau, la Universidad de Buenos Aires ya ha acreditado más de 234 posgrados y 10 carreras de grado, algunas de las cuales ya se han presentado en la segunda convocatoria de acreditación. Sin

| Panorama
| pp.33-44
| Volumen 10
| Número 19
| Julio-diciembre
| 2016
| 41


embargo, no ha encarado una evaluación institucional integral.

María Catalina Nosiglia I Verónica Mulle I A partir de algunos cambios recientes, podemos sostener que en la actualidad asistimos a un cuarto periodo en el que las universidades, en el seno del Consejo Interuniversitario Nacional, han comenzado a discutir algunos aspectos de la acreditación de carreras de grado, específicamente los criterios para definir cuáles de estas deben ser incluidas en la nómina de acreditación por cuanto las actividades que desarrollan sus graduados suponen riesgo social. Asimismo, se están debatiendo criterios para la formulación de estándares de acreditación, pues se propone generar efectos sobre las carreras y sus corporaciones.

Otro elemento que no es menor remite al surgimiento de procesos paralelos de evaluación de algunas funciones de las universidades, como es el caso de la evaluación de la investigación promovida desde el Ministerio de Ciencia y Tecnología. Esta iniciativa pone en cuestión el sentido holístico de la evaluación institucional de las universidades.

\section{REFLEXIONES FINALES}

A lo largo de estas páginas, se plantearon algunas reflexiones acerca de un conjunto de tensiones que observamos en las políticas de evaluación universitaria. Las cuestiones que se trataron se consideran puntos centrales en el contexto de las actuales políticas de educación superior. Las preocupaciones en torno a la calidad, la pertinencia, el planeamiento y la gestión eficaz y transparente, el ejercicio de la autonomía, forman parte de lo que Tunnermann (2010) ha definido como un "nuevo ethos académico" creado dentro de los recientes procesos de transformación universitaria.

Panorama I pp. 33-44 । Volumen 10 I
Tyler y Bernasconi (1999) advierten que debe tenerse presente

que el aumento en la calidad es un fenómeno de largo plazo, que depende de la voluntad y capacidad de las instituciones de mejorar, no del sistema de evaluación. En el mejor de los casos, éste crea las condiciones para que las universidades alcancen excelencia, las estimula, orienta, presiona, recompensa o castiga, para que no cejen en su esfuerzo, pero el sistema no puede mejorar a las instituciones sin su colaboración [...] los mecanismos de evaluación que ignoran que la calidad de la educación superior del país depende de la calidad de cada una de las instituciones que lo componen, están destinados al fracaso (1999, pp. 13-14).

A partir de estas reflexiones, consideramos que es necesario producir en las instituciones y en el conjunto del sistema universitario investigaciones en torno a las siguientes cuestiones: ¿en qué medida la evaluación institucional fue incorporada como una práctica sistemática, permanente y necesaria para el fortalecimiento de la gestión académica e institucional de las universidades? ¿De qué modo es usada la información producida por los procesos de evaluación y acreditación para la formulación de políticas institucionales y del conjunto del sistema? ¿En qué medida los procesos de evaluación siguen aportando elementos nuevos al mejoramiento institucional o se han tornado prácticas burocratizadas?

Por otra parte, teniendo en cuenta la mirada del planeamiento institucional, y la calidad como concepto relativo, ¿es posible pensar en modelos e instrumentos uniformes de evaluación dadas las diferencias entre las misiones, funciones y dimensiones de las instituciones?

La evaluación de la calidad probablemente ha sido el eje de las políticas del sector universitario en las últimas décadas en el ámbito regional y en especial en el contexto de Argentina. Según Dias Sobrinho (2007), los procedimientos llevados adelante por los Gobiernos y las agencias multilaterales han tendido a definir la calidad como la demostración mensurable de desempeños en la graduación de profesionales, los rendimientos estudiantiles, la producción científica de los académicos $\mathrm{y}$ otros productos que pueden ser objetivamente cuantificados. Sin embargo, no se ha interrogado sobre los sentidos y valores (científicos, éticos, sociales, etc.) de las ideas, las misiones, las visiones, los proyectos, el proceso, los impactos relaciones llevados a cabo en las instituciones y los sistemas.

Siguiendo al autor, "no habiendo interrogación sobre los valores, es decir, producción de sentidos, los sistemas de evaluación acaban practicando solo una cuasievaluación, no propiamente una evaluación" (Dias Sobrinho, 2007, p. 322). Como señalamos para el caso argentino, estas prácticas se transformaron inicialmente en procesos de control más que de evaluación. Y sumado a ello, se 
multiplicaron los enfoques y mecanismos de evaluación: la evaluación institucional, la acreditación de carreras de grado y posgrado, la evaluación de docentes investigadores - a través de un programa específico- y la evaluación de la función investigación a cargo de otro organismo. En este sentido, cabe preguntarse ¿cómo sobrevivirá la evaluación institucional ante la emergencia de los rankings y de los mecanismos de evaluación llevados a cabo por otras agencias interesadas en aspectos particulares de las funciones universitarias? ¿Existe una sobrecarga de instancias de evaluación que provoca resistencias entre los evaluados y disminución de evaluadores dispuestos a realizar esta función?

Por último, desde una mirada prospectiva, ¿el balance y los desafíos en el futuro de la evaluación de la calidad universitaria están —o deberían estar- en la agenda de debate de las políticas universitarias?

Estos interrogantes, esbozados a modo de cierre, se proponen como temas de debate dentro de los espacios de decisión de políticas universitarias o hipótesis para futuras investigaciones. Su consideración plantea cuestiones para analizar y proponer mejoras al sistema de evaluación y acreditación de la calidad universitaria, así como futuros horizontes para la investigación y la gestión política de estos procesos, en los que las propias instituciones universitarias públicas — dentro de su autonomía - cumplen un rol fundamental, que es preciso desarrollar con un compromiso activo.

\section{REFERENCIAS}

1. Araújo, S. (2015). Evaluación universitaria: dos enfoques, dos dinámicas. Revista Politicas Universitarias, 2, 26-31.

2. Argentina, Ley de Educación Superior 24521 de 1995 (10 agosto 1995).

3. Argentina, Juzgado Primera Instancia en lo Contencioso Administrativo Federal N 1. "UBA c/Estado Nacional”, sentencia del 19/02/1996.

4. Argentina, Secretaría de Políticas Universitarias del Ministerio de Educación de la Nación (2014). Anuario de estadísticas universitarias 2012. Buenos Aires.

5. Argentina, Dirección Nacional de Información y Evaluación de la Calidad Educativa, Ministerio de Educación de la Nación (2015). Anuario estadístico educativo 2014. Buenos Aires.
6. Argentina, Comisión Nacional de Evaluación y Acreditación Universitaria (Coneau) (2015). Guía de posgrados acreditados año 2014. Recuperado de http://www.coneau. gov.ar/archivos/publicaciones/documentos/ PosgradosAcreditadosRA2015.pdf

7. Brunner,J. (1993). Evaluación y financiamiento de la educación superior en América Latina: bases para un nuevo contrato. En H. Courard (ed.), Políticas comparadas de educación superior en América Latina. Santiago de Chile: FLACSO.

8. Brunner, J.y Villalobos, C. (2014). Políticas de educación superior en Iberoamérica, 20092013. Ponencia presentada en III Encuentro de Rectores Universia 2014, Río de Janeiro.

9. Camilloni, A. (3 diciembre 2004). Los procesos de autoevaluación en la educación superior. Conferencia presentada en la Facultad de Farmacia y Bioquímica de la Universidad de Buenos Aires. Mimeo.

10. CONEAU (2015). Informe de autoevaluación. Recuperado de: http://www.coneau.gov.ar/ CONEAU/wp-content/uploads/2015/09/ CONEAU-Informe-de-Autoevaluación-20151. pdf

11. De la Fare, M. y Lenz, S. (2010). La politica de posgrado en la Argentina y la expansión de carreras. Buenos Aires.

12. Dias Sobrinho,J. (2012). Políticas y conceptos de calidad: dilemas y retos. Avaliação, 17(3), 601-618.

13. Dias Sobrinho, J. (2003). Avaliação da educação superior regulação e emancipação. Avaliação, $8(2), 31-47$.

14. Dias Sobrinho, J. (2007). Evaluación de la educación superior en Brasil: la cuestión de la calidad. En P. Krotsch, A. Camou y M. Prati, Evaluando la evaluación: politicas universitarias, instituciones y actores en Argentina y América Latina (pp. 311-329). Buenos Aires: Prometeo.

15. Dobbins, M., Knill, C.y Vögtle, E. M. (2011). An analytical framework for the cross-country comparison of higher education governance. Higher Education, 62(5), 665-683.

16. Espinoza D., O., González F., L. E., Poblete L., Á., Ramírez G., S., Silva T., M.y Zúñiga C., M. (1994). Manual de autoevaluación para instituciones de educación superior:pautas y procedimientos. Santiago de Chile: Centro Interuniversitario de Desarrollo. Recuperado de http://www.cinda.c1/download/libros/1-Manual \%20Autoevaluaci \%C3 \%B3n \%20

para \%20Instituciones \%20de \%20

Educaci \%C3 \%B3n \%20Superior.pdf 
17. Fernández Lamarra, N. (2007). Educación superior y calidad en América Latina y Argentina: los procesos de evaluación y acreditación. Buenos Aires: EDUNTREF.

María Catalina

Nosiglia I

Verónica

Mulle I

18. Follari, R. (2014). Autonomía versus planificación estatal: hacia una superación de la dicotomía. En R. Follari, A. Stubrin, A. Camou y M. Marquina, La universidad entre la autonomía y la planificación: tres ensayos en diálogo. Buenos Aires: Instituto de Estudios y Capacitación, Federación Nacional de Docentes Universitarios.

19. Kretek, P. M., Dragšić, Ž. y Kehm, B. M. (2013). Transformation of university governance: on the role of university board members. Higher Education, 65(1), 39-58.

20. Krotsch, P. (2001). El proceso de formación e implementación de las políticas de evaluación de la calidad en Argentina. En A. Chiroleu, Repensando la educación superior. Rosario, Argentina: UNR Editora.

21. Marquina, M. (2009). Académicos como evaluadores de instituciones y carreras universitarias en Argentina: la experiencia de una década. En M. Marquina, C. Mazzola y G. Soprano, Politicas, instituciones y protagonistas de la universidad argentina. Buenos Aires: Prometeo.

22. Mollis, M. (1999). El campo de la evaluación universitaria argentina y los organismos internacionales: entre la autonomía y la heteronomía. Perfiles Educativos, 84, 22-36.

23. Naidorf, J., Giordana, P. y Horn, M. (2007). La pertinencia social de la universidad como categoría equívoca. Nómadas, 27, 22-33.

24. Neave, G.y Van Vught, F. (1991). Prometeo encadenado: Estado y educación superior en Europa. Barcelona: Gedisa.

25. Nosiglia, M. C. (2008). Entre normas: continuidades y rupturas de las orientaciones políticas en materia de regulación de la educación superior. En Actas del III Encontro Internacional de Pesquisadores de Politicas Educativas (pp. 35-52). Porto Alegre: Universidade Federal do Rio Grande do Sul.

Panorama I

pp. 33-44 I

Volumen 10 I

Número 19 |

Julio-diciembre

2016 |

27. Stubrin, A. (2014). Autonomía universitaria, planeamiento y política pública: un ensamble factible e indispensable. En R. Follari, A. Stubrin, A. Camou y M. Marquina, La universidad entre la autonomía y la planificación: tres ensayos en diálogo. Buenos Aires: Instituto de Estudios y Capacitación, Federación Nacional de Docentes Universitarios.

26. Nosiglia, M. C. (2013). Universidad y evaluación de la calidad, una relación controvertida: el caso de la UBA. En M. C. Nosiglia (comp.), La evaluación universitaria: reflexiones teóricas y experiencias a nivel internacional y nacional (pp. 191-204). Buenos Aires: EUDEBA.
28. Tunnermann, C. (2010). Pertinencia y calidad en educación superior. En Fernández Lamarra, N. (comp.), Universidad, sociedad e innovación. Buenos Aires: EDUNTREF.

29. Tyler, L. A. y Bernasconi, A. (1999). Evaluación de la educación superior en América Latina: tres órdenes de magnitud. Cambridge, MA: Harvard University.

30. Unesco (1998). Conferencia Mundial de la Educación Superior. París.

31. Unzué, M. (2014). Pertinencia, calidad y evaluación: potencialidades y límites de una relación compleja. En R. San Martín (ed.), Evaluación y acreditación universitaria: actores y politicas en perspectiva. Buenos Aires: Universidad de Palermo.

32. Unzué, M. (2015). La compleja articulación entre políticas públicas y universidad. Revista Politicas Universitarias, 2, 22-25. 